\title{
Perceiving Layered Information on 3D Displays Using Binocular Disparity
}

\author{
Nora Broy \\ BMW Group Research and Technology \\ Nora.NB.Broy@bmw.de
}

\author{
Florian Alt, Stefan Schneegass, \\ Niels Henze, Albrecht Schmidt \\ University of Stuttgart \\ \{firstname.lastname\}@vis.uni- \\ stuttgart.de
}

\begin{abstract}
3D displays are hitting the mass market. They are integrated in consumer TVs, notebooks, and mobile phones and are mainly used for virtual reality as well as video content. We see large potential in using depth also for structuring information. Our specific use case is 3D displays integrated in cars. The capabilities of such displays could be used to present relevant information to the driver in a fast and easy-to-understand way, e.g., by functionality-based clustering. However, excessive parallaxes can cause discomfort and in turn negatively influence the primary driving task. This requires a reasonable choice of parallax boundaries. The contribution of this paper is twofold. First, we identify the comfort zone when perceiving 3D content. Second, we determine a minimum depth distance between objects that still enables users to quickly and accurately separate the two depth planes. The results yield that in terms of task completion time the optimum distance from screen level is up to 35.9 arc-min angular disparity behind the screen plane. A distance of at least 2.7 arc-min difference in angular disparity between the objects significantly decreases time for layer separation. Based on the results we derive design implications.
\end{abstract}

\section{Categories and Subject Descriptors}

H.5.2 [Information Interfaces and Presentation]: User InterfacesScreen design (e.g., text, graphics, color)

\section{General Terms}

Human Factors

\section{Keywords}

3D displays; human factors; automotive user interfaces

\section{INTRODUCTION}

The past years saw a significant drop in prices for 3D display hardware. As a result, many consumer devices are equipped with displays that allow depth information to be exploited. TVs enable

Permission to make digital or hard copies of part or all of this work for personal or classroom use is granted without fee provided that copies are not made or distributed for profit or commercial advantage and that copies bear this notice and the full citation on the first page. Copyrights for components of this work owned by others than ACM must be honored Abstracting with credit is permitted. To copy otherwise, to republish, to post on servers or to redistribute to lists, requires prior specific permission and/or a fee.

PerDis '13, June 04 - 05 2013, Mountain View, CA, USA Copyright 2013 ACM 978-1-4503-2096-2/13/06...\$15.00.

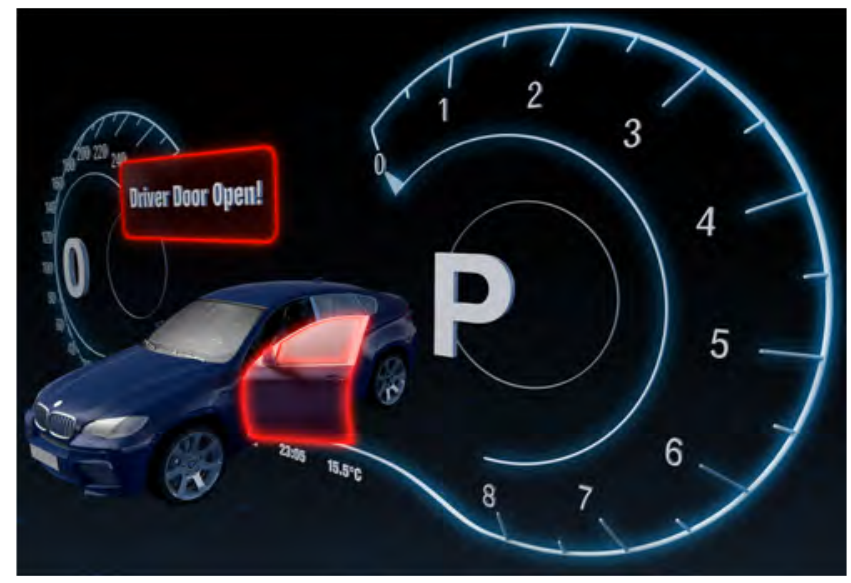

Figure 1: Examplary illustration of using 3D to highlight important information in the car. While information such as time, temperature, and trip distance remain in the background, a pointer to the open door is shown in the foreground.

watching 3D movies, laptops allow for playing 3D games, and mobile phones can be used to create and show 3D images or clips. Today, the vast majority of 3D content consists of virtual reality in games and images of the physical world in 3D photos or movies. However, advances in display hardware make us believe that in the near future novel application areas for 3D displays will emerge. Whereas active shutter, polarization, or anaglyph 3D requires the user to wear glasses, autostereoscopic displays are readily usable without wearing additional gear. We believe that such displays will soon be found in cars - not only to entertain the passenger, but more importantly to cluster informative content. Depth information can be used to structure content for enhancing perception and information processing. In this way, we envision navigation cues to be presented in an easy-to-understand manner, e.g., for judging the distance to the next turn action. Further application scenarios include displaying warning information on the car status, e.g., that a door is open (cf. Figure 1) or that refueling is required soon. This information could poke out of the screen as the user's attention is required, while currently less important information could be displayed further in the back.

The main problem with such applications is that moving objects away from the screen layer, which people are used to from 2D displays, can cause discomfort with increasing distance. This is less of a problem for entertaining content compared to scenarios where users are potentially engaged in another primary task, for example driving. In addition, users need to be able to distinguish quickly and accurately, which content is displayed in the front/back. 
To lay the foundation for creating usable 3D information interfaces the contribution of our work is twofold. First, we aim to identify a comfort zone, i.e., the distance from the screen plane (to the back and to the front) in which users can comfortably perceive the content. Second, we aim to investigate the optimum depth distance between two objects so that users can distinguish the object displayed in front from the object displayed in the back as quickly (task completion time) and accurately (error rate) as possible.

We report on two user studies. The initial study with 21 participants yields that the comfort zone strongly depends on the user but for the majority of participants lies within 36 arc-min of angular parallax. In a subsequent study with 18 users that investigates the optimum depth distance between two objects we found that (a) accuracy is highest for an angular disparity difference of more than 2.7 arc-min, and that (b) task completion time is optimal if the two objects are positioned between zero parallax up to 36 arc-min of angular disparity behind the screen plane.

\section{BACKGROUND AND RELATED WORK}

The following section provides background information relevant to our work. First, we briefly introduce different 3D technologies, before focusing on potential application domains. We then address related work with regard to human factors of stereoscopic displays.

\subsection{Technology}

A wide variety of technologies exists that enables $3 \mathrm{D}$ vision. Stereoscopic displays create a 3D effect through binocular disparity, showing the images for the left and right eye with either time multiplexed (e.g., shutter glasses) or time parallel (e.g., polarization glasses) technology [15]. One of the main drawbacks of these technologies is the use of glasses, which makes it difficult to be used in public settings or while driving a car. Autostereoscopic displays, i.e., displays without the need for additional equipment, can partially overcome this problem, but are still limited to single users. Multi-stereoscopic displays, i.e., displays that allow stereoscopic images to be seen from any viewpoint without glasses, are still in their infancy but hold great promise for the future [16]. Further technologies that make using glasses obsolete include volumetric displays. For an overview we refer to the work of Favalora [4]. As volumetric displays require a considerable amount of space we focus and draw from prior work on stereoscopic displays, being the most appropriate technology for our use case.

The visualization of 3D images on a 2D plane (e.g., a display) needs depth cues to simulate the third dimension. Prior work has identified a number of cues, including shadows, occlusion, perspective, and binocular disparity. Cutting [3] provides an in-depth investigation of which depth cues are suitable for which distances. Occlusion and relative size are very strong cues that work at arbitrary distances. However, the possible occlusion of objects limits their use for cases, where the information of the object (e.g., text) is important. Similarly, relative size may render content unreadable or unrecognizable if displayed too far away from the user. We found binocular disparity to be particularly suitable in our case, as it works well for small and large distances between objects while at the same time preserving the perceptibility of content.

\subsection{Application of 3D Displays}

Nowadays, 3D displays are mostly used in the entertainment sector. The vast majority of televisions come along with integrated 3D functionality. Furthermore, the gaming industry is rapidly picking up on displays being widely available. Research in this area is mainly concerned with user experience (UX). Schild et al. investigate the effect of 3D in video games on UX [11]. They show that the
UX improves using 3D displays compared to conventional 2D displays. Additionally, Litwiller and LaViola point out that the game performance is slightly better with $3 \mathrm{D}$ vision [9]. Note, that they only tested games developed for virtual 3D on 2D displays and, therefore, did not take the increased design space into account.

The benefit of using 3D for visualizing (complex) content has been demonstrated in many domains such as in medicine [14] or graph visualization [1]. The use of 3D displays in the automotive domain has been investigated by Broy et al. [2], who identified benefits regarding attractiveness when visualizing an in-vehicle information system's menu structure on a 3D display, compared to $2 \mathrm{D}$ displays. In contrast, we look at where and how to best present information to increase user performance.

\subsection{Human Factors}

Binocular disparity is using different images for both eyes. Shifting the position of an object on one of the images leads to a threedimensional effect [15]. Julesz first created 3D images using random dot images [7]. He only used binocular disparity, showing that users can perceive depth in images that contain no further depth information. Froner et al. looked into displaying different levels of disparity using only binocular disparity as a depth cue [5]. They compared seven displays showing two white squares on a black background with one of them having $0,1,2$, or 3 pixels disparity. Through categorizing the display types in three classes they found differences in task performance in terms of error rates due to the used display technology. In contrast to this work, we investigate task completion times within the comfort zone.

An important issue when creating stereoscopic 3D images is the level of comfort users feel when looking at 3D images. One of the most important reasons for visual discomfort is that a large parallax is more difficult to fuse $[8,13]$. Therefore, it is important to use an appropriate parallax. Jones et al. investigated how far virtual objects can be moved within the virtual space [6]. They found out that there are boundaries in which users were capable of fusing the images. This boundaries strongly vary between different users. Furthermore, they found that when people look away from the scene - as is the case when people focus on traffic at the same time - it is much harder to fuse the images. Quintus and Halle developed a composition tool that can be used for creating comfortable stereoscopic images [10]. The tool visualizes the appropriate depth layers in which users do not feel visual discomfort.

In our research we aim to draw a comprehensive picture of how $3 \mathrm{D}$ information can be visualized in an intuitive and easy-to-understand manner. Whereas previous work focuses on particular (technical) issues we define the comfort zone and assess user performance by measuring task completion time, error rates, and the users' subjective feedback.

\section{PROTOTYPE}

We built a prototype that allows us to position objects in $3 \mathrm{D}$ space. The idea behind the design is to simulate a situation in which only binocular disparity would be used to structure information in 3D space. In our initial work, we focus on the most simple use case of showing two distinct objects on the screen, drawing upon prior work by Froner et al. [5]. To minimize any influence of content, our prototype shows two squares of equal size positioned next to each other in the middle of the $3 \mathrm{D}$ display and at zero parallax. The distance between the inner edges of the two squares amounts 109 pixels. The width of the squares is 131 pixels each. Arbitrary textures can be added to the squares. For the purpose of the study, the z-position of each of the squares is altered in discrete steps - either explicitly using the keyboard, or automatically by using a script. 
We choose the smallest possible step size through specifying parallax sizes in pixels. For our setup (pixel pitch $=0.196 \mathrm{~mm}$; viewing distance $=750 \mathrm{~mm}$ ) -1 pixel parallax corresponds to 54 arc-sec of angular disparity, resulting in a perceived depth of $2.3 \mathrm{~mm}$ in front of the screen. Froner et al. [5] describe the conversions between pixel parallax, angular disparity, and vertex depth from screen. Negative pixel values corresponds to positions in front of the screen and positive values to locations behind the screen.

As we aim to isolate the effect of binocular disparity on viewing comfort for layered information presentation the application eliminates other depth cues. We avoid any occlusion and ignore relative size through maintaining the initial size of the objects on the screen even if they are moved along the z-axis. This creates the impression of shrinking objects as they move towards the user and of growing objects as they move away. Through this exclusion of monocular cues the 2D representation of the task shows no visible depth effect.

The prototype is running on an Asus G75VW notebook with a stereoscopic 3D screen. The system uses the Nvidia 3D Vision 2 shutter technology to present scenes stereoscopically. The notebook's display has a screen size of 17" with a resolution of $1920 \mathrm{x}$ 1080 pixels. Since we aim at smaller screens, that are commonly used for the automotive domain we just rendered the tasks on a centered area of the display with a resolution of $1280 \times 480$ pixels. The software was implemented using the game development engine Unity3D with $\mathrm{C \#}$ as scripting language.

\section{DETERMINING THE COMFORT ZONE}

Excessive parallaxes reduce the viewing comfort [8]. However, suggested limits for parallax reported in previous work strongly vary $[5,6,10]$. Therefore, we determine the zone in which binocular disparity is perceived as comfortable for our setup. In this study, we identify the maximum distances from the screen at which it is still comfortable to perceive a content element (fusing the images for both eyes). We asked participants to define their individual comfort zone by letting them alter the z-position of objects.

\subsection{Apparatus and Experimental Setup}

Participants used the prototype described above. They altered the z-position of both or just one square. Two buttons were used to move the squares forward and backward. By pressing the space bar the position was confirmed and the system recorded the disparity in pixels. Participants were seated in front of the system at a distance of $75 \mathrm{~cm}$ - the typical distance between driver and the instrument cluster. A chin rest ensured a constant distance to the display.

\subsection{Study Design}

We used a repeated measures design to determine the maximal disparity still perceived as comfortable. The independent variables are content, direction, and number of focus layers:

Content: As we envisioned a potential effect based on the presented content, we tested plain squares and squares textured with arrows, as can be found in a navigation system.

Direction: Starting from the screen plane, we were interested in how far the comfort zone stretches to the front and to the back of the display.

Number of focus layers: Information on 3D displays can be presented on different layers. As users can only focus on one layer at a time we wanted to investigate the impact of a different number of focus layers. We distinguish between shifting both squares at once and shifting just one of them while the other remains at the initial position (zero parallax).
Each independent variable has two levels, resulting in eight conditions. We did not expect sequence effects through the order in which direction and the number of focus layers are assessed. Therefore, we only counterbalanced the order of the content presentation. Half of the participants started with the plain squares and the other half with the squares containing an arrow.

\subsection{Procedure}

Participants were recruited through our internal mailing lists. As participants arrived, we provided them a brief introduction to stereoscopy and explained them the course of the study. Participants first completed a stereo vision test based on Julesz's Random Dot Stereograms (RDSs) [7]. The stereo vision test consists of 8 RDSs that depict different shapes appearing in front of the display. The parallax of the shapes was set to -1 pixel. The participants were then asked to identify the hidden shapes. If a participant recognized less than six of the eight presented RDSs correctly, they were excluded from the study. After participants successfully passed the stereo vision test, the tasks for assessing the comfort zone started.

In the first task, the participants were asked to use two buttons on the keyboard to move both squares from the screen level to the front in discrete steps. The participants had to find the maximum distance of the squares from screen depth that is still comfortable to fuse. The participants were instructed to ensure the selected depth level by averting the eyes from the screen for several seconds and focusing on the squares again. If the refocusing was perceived as comfortable they had to confirm the depth position by hitting the space bar. After that, we aimed to find the most comfortable depth position backwards. Starting again with both squares being shown on the screen level, participants were required to move the squares to the back and to tell us until when this is still perceived to be comfortable. There was no time limit for completing the task.

In the second task, participants were asked to move one square from the screen level to the front while the other square remained on the screen level. Again the participants adjust the maximum disparity still comfortable to fuse and confirms the value by focusing the displayed stimulus again after averting the eyes from the screen. By hitting the space bar the position was recorded. The procedure was then repeated for the right square. No time limit applied.

After that, the participant repeated the first as well as the second task, but this time the displayed squares were textured with arrows. All participants completed all conditions with a counterbalanced order of the content conditions.

After completing all tasks, they were asked about the difficulty for adjusting comfortable depth levels and about symptoms like headache, eye strain, or motion sickness. In total the study took roughly 20 minutes per participant.

\subsection{Results}

21 participants (\%: 7; $0^{7}: 12$ ) aged between 22 and 53 years $(M=31.4, S D=9.4)$ took part in the study. We excluded 2 participants from the study. One recognized less than 6 out of the 8 presented RDS and one achieved extreme parallax values due to extreme shortsightedness.

We determined the average disparity that is perceived as comfortable for all conditions. Moving both plain squares to the front, the average disparity is -168.9 pixels $(S D=106.3)$, and moving them to the back the average disparity is 217.1 pixels $(S D=98.0)$. The mean disparity is -67.2 pixels $(S D=36.0$ ) when moving just one of the plain squares to the front and 72.9 pixels $(S D=35.2)$ when moving one plain square to the back. For the textured squares, the average disparity is -207.5 pixels $(S D=137.1)$ if both are moved to the front and 193.3 pixels $(S D=102.1)$ if both are moved to the 


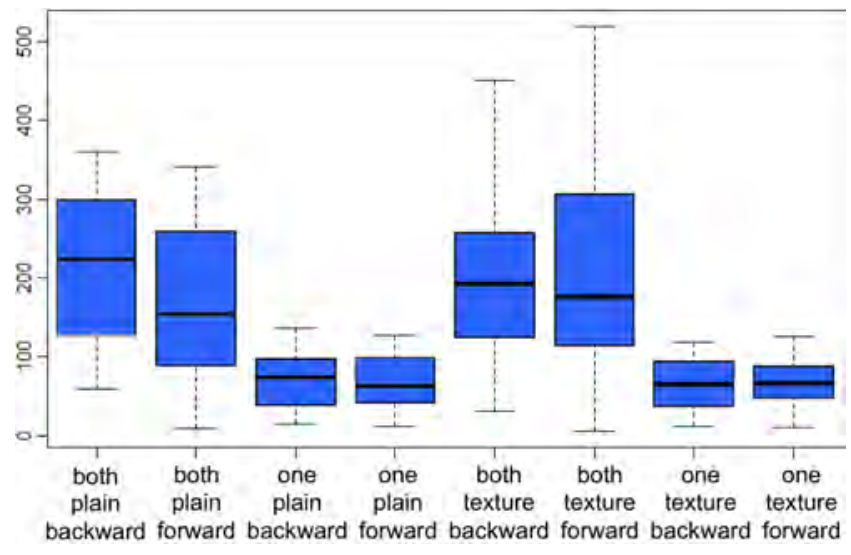

Figure 2: Box-Whisker-Diagramm of the parallaxes in pixels rated as comfortable.

back. Moving just one textured square to the front results in -66.0 pixels $(S D=32.8)$ disparity and moving one textured square to the back results in 64.4 pixels $(S D=34.7)$ disparity. The box plot in Figure 2 depicts the high variances in all conditions.

A Kolmogorov-Smirnov test shows that the data is normally distributed. Thus, we conducted a repeated measures analysis of variance (ANOVA). The ANOVA shows that adjusting only one square results in a significantly lower disparity compared to shifting both squares, $F(1,21)=40.308, p<.001$. The other main effects as well as the interaction effects are not significant, all $p>.05$.

Overall, the results reveal a very high variance between the participants. Moving one square results in a lower variance and also in a smaller comfort zone. Although the subjects experienced depth values beyond their individual comfort zone, no symptoms associated to visual discomfort and fatigue are reported in the conducted interviews. Nine participants spontaneously mentioned that the tasks with textured squares are easier than with non-textured squares.

\subsection{Discussion}

The study revealed a significant difference if only one square is moved and the other remains at a fixed position instead of moving both squares. The very high variance is reflected by the high standard deviations. We likely found no other significant effects because of high intra- and intersubject variance. The large variance indicates that the comfort zone highly varies for each person and thus depends on individual features. These features may be of psychological or physiological nature. For instance, the individual comfort zone can be influenced by individual interaxial distances (eye separation), visual acuity, and prior experiences with stereoscopy.

Adjusting one square decreases the variances. Since one square stays at screen level while the comfort zone is defined via the other square, we assume that a reference object at screen depth makes it easier for participants to assess the depth range and also reduces the comfort zone. Systems that use binocular disparity for information presentation must therefore either enable users to define their individual comfort zone or use conservative limits.

The results show similar parallax limits at the front and at the back. We assume that the comfort zone is symmetrical and positive parallaxes may be applied in the same manner as negative parallaxes. We investigate this in more detail in a follow-up study.

A conservatively chosen comfort zone ranges from -40 to 40 pixels parallax (35.9 arc-min angular disparity). In total, $75 \%$ of the participants perceived this zone as comfortable for textured squares on two focus layers. Therefore, we use this as the maximum depth zone for the second study. We apply textured squares as stimuli.

\section{DEPTH AND PERFORMANCE}

While we identified the boundaries of the comfort zone in the first study, we more closely investigate the effect of depth perception on user performance in the second study. Since task completion time has to be minimized for automotive applications to reduce the influence on the primary driving task, we are interested to identify an area inside the comfort zone which maximizes performance in separating two depth layers. Particularly, we investigate how (a) the distance between two objects at (b) different positions within the comfort zone would impact on task completion time and error rate. We tested 4 distances (1, 2, 3, and 4 pixels) at five positions $(-40,-20,0,20,40$ pixels, 0 pixels being the screen level).

\subsection{Apparatus and Study Design}

We again used the prototype described in Section 3 to display two squares on the screen. This time, the distance was not controlled by the user, but we used a script to present the stimulus on the screen. The user's task was to decide as quickly and as accurately as possible, which square was closer to the user. The study is designed as a repeated measures experiment with the following independent variables:

Position: Based on the previous findings we tested different positions of content linearly distributed over the comfort zone at $-40,-20,0,20$, and 40 pixels from the screen.

Depth Difference: To determine the optimum distance, we present two squares with varying depth differences at each position. The difference between the objects' parallaxes varies between $1,2,3$, and 4 pixels. We used every possible combination (e.g., left 1 pixel to the front and right 2 pixels to the back for a 3 pixels depth difference) and balanced all conditions.

This results in a total of 20 conditions and 80 stimuli that were presented in a randomized order. As content we used squares textured with arrows. We measured task completion time (i.e., the time between presenting the stimuli and the user making the decision which square is presented further at the front) and error rates.

\subsection{Experimental Setup and Procedure}

Similar to the first study, participants were provided a brief introduction to stereoscopy and a brief overview of the study as they arrived at the lab. In order to qualify for the study they had to pass the stereo vision test, that we had already applied for the first study (see Section 4.3), as well as a Snellen test [12], which measures visual acuity. Participants who passed the test then proceeded with the main task. They were seated $75 \mathrm{~cm}$ in front of a 3D display. A chin rest was used again to maintain the distance between test person and screen. A keyboard with two keys was provided - one representing the left square and one representing the right square. Participants were then asked to press the button for the square that appeared closer to them. There was no time limit but participants were asked to be as quickly and as accurately as possible. Then the 80 stimuli were presented in random order. After each stimulus we showed visual noise on the 3D display while the participants solved a distractor task on a TV screen in order to force accommodation switches. Therefore, we placed the TV screen 2 meters in front of the subject's position behind the stereoscopic display. The TV screen showed words composed of two simple, unrelated nouns (e.g., kiwi-earring, fir-water). Participants were asked to read out the word shown on the display aloud. After that, pressing either of the two keys triggered the next stimuli to be shown after $500 \mathrm{~ms}$ in order to guarantee that users were already focused on the $3 \mathrm{D}$ display when the stimulus appeared and timing started. 


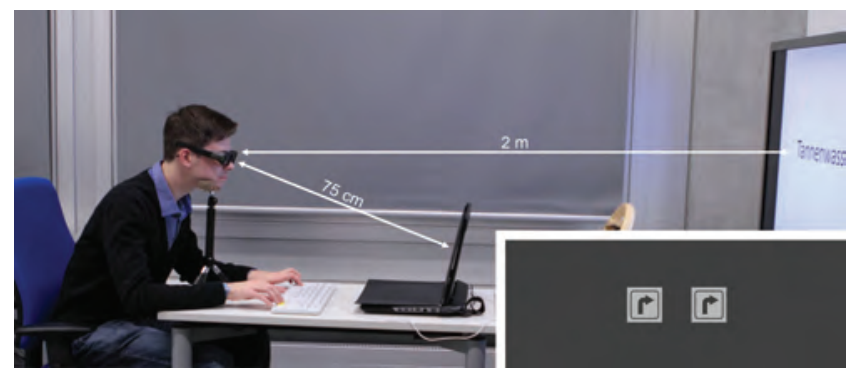

Figure 3: Study Setup - Participants were positioned at $75 \mathrm{~cm}$ in front of the stereoscopic display and at $2 \mathrm{~m}$ from the screen showing the distractor task. In the lower right corner the stimulus is shown.

To minimize learning effects, a set of sixteen randomly chosen stimuli was presented in the beginning to each subject before seamlessly starting to show the eighty stimuli we prepared. Short breaks were taken after every 16 th stimulus. At the end we conducted semistructured interviews with the participants. We were particularly interested in the difficulty of the task and the personal experience with the 3D effect with regard to visual fatigue and discomfort.

\subsection{Results}

In total, 18 participants ( $\left(\right.$ : $\left.4 ; \sigma^{7}: 14\right)$ aged 20 to 31 years $(M=$ $25.4, S D=2.9$ ) completed the study. None of them participated in the first study. All subjects had corrected to normal visual acuity and had no problems in recognizing the -1 pixel RDSs.

A Kolmogorov-Smirnov test reveals no normal distribution of the data, $p<.05$. Hence, we used non parametric tests for the statistical analysis. Since to the best of our knowledge no nonparametric methods for a two-way ANOVA with repeated measures exists, we analyzed the main effects with Friedman tests.

For depth difference we could not find any statistically significant effects in the correctness of the answers, $X^{2}(3)=7.538$, $p=.057$. But there is a significant effect for task completion time, $X^{2}(3)=34.2, p<.001$. We performed a post hoc analysis by means of Wilcoxon Signed Rank tests using a Bonferroni correction to adjust the error levels. All pair-wise comparisons reveal significant effects, $p<.005$, except the comparison of 3 and 4 pixel disparity, $p=.472$. Figure 4 shows the means and standard errors for the depth differences in parallaxes between the displayed squares. The plot reveals that the means decrease with increasing distance between front and back square.

Means and error rates depicting the performance on the five different positions in the defined comfort zone are shown in Figure 5. Similar to the depth distances between the squares, the variation of the reference layer in depth is not significant for errors, $X^{2}(4)=$ $0.232, p=.994$, but for task completion time, $X^{2}(4)=36.978$, $p<.001$. Wilcoxon tests with a Holm-Bonferroni correction show that for the foremost position of -40 pixels parallax reaction times are significantly higher compared to all other conditions, $p<.007$. At 20 pixel disparity, the task completion time is significantly lower compared to the -20 parallax condition, $p=.002$.

The interviews revealed that none of the participants felt uncomfortable in terms of visual fatigue and symptoms like headache, motion sickness, or eyestrain. Eight participants considered some of the stimuli to be more difficult than others. It seems that this is a result of the different depth distances between the objects. Four participants stated that the very front positions of the squares were more difficult and one pointed out that stimuli consisting of squares presented at larger distances from the screen were more demanding. Overall, the participants rated the task as not arduous.
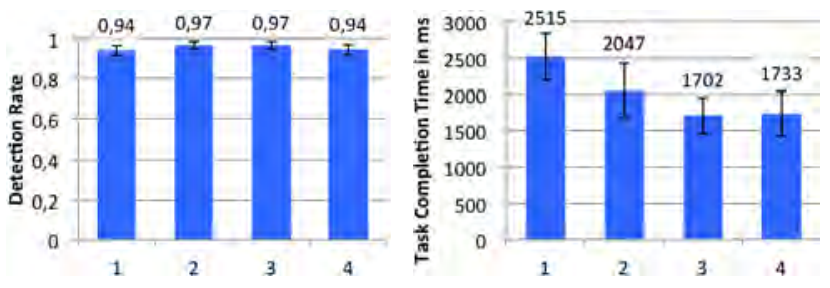

Figure 4: Mean values and standard errors as error bars for parallax differences of $1,2,3$, and 4 pixels. The means of task completion time decrease with increasing distance between front and back square and are lowest for 3 or more pixels.


Figure 5: Mean values and standard errors as error bar for depth positions of $-40,-20,0,20$, and 40 parallax in pixels. Whereas the detection rate is constantly high over all conditions, task completion time is lowest at $\mathbf{- 2 0}$ pixels.

\subsection{Discussion}

The results show an overall low error rate when solving the task. This is a strong indicator that binocular disparity enables an accurate depth perception - even if other depth cues are excluded. Furthermore, the impression of depth is not negatively affected by the constant size of the content. These findings are valuable for user interface design, particularly for small display sizes. The reason is that an accurate depth impression can be achieved without the need to shrink the content, which would make it unrecognizable or unreadable. In addition, objects that are presented in the foreground do not need to become larger and thus occupy valuable space for visualizing further information or occluding other objects on the screen.

The low error rate suggests that small depth differences can be accurately recognized. Additionally, as the $z$ distance between the objects increases participant's information processing and perception occurs faster. Our findings suggest that 3 pixels parallax difference (corresponding to 2.7 arc-min angular disparity at screen depth) is a threshold beyond which no more significant increase in task completion time is expectable.

Within the comfort zone, there is a number of indications that it is easy for the users to solve tasks with high accuracy. This is reflected by the low error rates and the fact that the tasks were rated as neither being demanding, nor uncomfortable, nor as causing visual fatigue. The findings suggest that the comfort zone could be narrowed down to decrease task completion time. No statistical differences could be shown within the area of 0 up to 40 pixels of positive parallax (35.9 arc-min angular disparity). The task completion time tends to be lowest for 20 pixel positive parallax (17.9 arc-min angular disparity). Moreover, our results show that task completion times are significantly higher for negative parallaxes than for positive parallaxes. 


\section{LIMITATIONS}

The study has several limitations. First, we tested just two types of content (squares with/without arrows) in a low complexity screen design. Arrows would naturally occur in automotive environments (e.g., for navigation) but are usually presented together with additional (textual) information. Hence, more complex screen designs including different types of content could be subject to future investigations. Second, the study is limited by a rather abstract task and future work should verify the results under driving conditions. By narrowing the focus of the experiments we hope to increase their reproducibility and allow results to be applied to a wide range of application areas.

\section{IMPLICATIONS}

Based on two studies we investigated the comfort zone for displaying 3D information and determined the impact of depth differences between objects on accuracy and task completion time. In general, our studies let us derive the following implications:

Allow people to define their individual comfort zone. Our first study revealed that the maximum range in which people feel comfortable to perceive 3D content strongly varies between the participants. As a result, systems that exploit 3D capabilities to present information should allow the user to determine and define a personal comfort zone in which information is presented. Alternatively, rather conservative values should be chosen.

Provide visual reference points at screen level. We found a decrease in the variance if one object remains at the screen layer as reference point. This suggests that for the vast majority of participants this location is highly comfortable and could be used as an anchor point for crucial information. More and less important information could then be aligned accordingly.

Binocular disparity allows highlighting important information. Despite the fact that a lot of different depth cues exist, binocular disparity is suitable for information representation as even occlusion or changes in size are neglected. At the same time we are able to attribute high accuracy and rather low task completion times for abstract information representations, making it particularly helpful for essential information.

Information should be displayed behind the screen. Interestingly, participants were significantly faster to judge the depth relationship between objects as they are presented behind the screen. As a result, we suggest to display information with positive parallaxes, while the use of negative parallaxes (positions in front of the screen) have to be applied carefully.

Maintain a minimum depth difference. In general, the distinction between different depth layers due to binocular disparity was shown to be very accurate. However, we found that a minimum of 2.7 arc-min leads to significantly better task completion times when it comes to distinguishing two different depth levels.

\section{CONCLUSION}

In this paper, we investigated how to best visualize layered information on 3D displays using binocular disparity. We first explored the so-called comfort zone in which users can easily perceive information. Then we identified the optimum distance from the screen and the optimum distance between two objects for which users can as quickly and as accurately as possible separate two depth layers.

We conducted our evaluation in an automotive-like setting, showing that the comfort zone is strongly user-specific and that information should optimally be displayed behind screen level with angular disparities of up to 35.9 arc-min. The distance between two depth layers should at least be 2.7 arc-min.
For future work we plan to investigate the influence of size/shape of the objects and of the vertical and horizontal distance between objects on accuracy and velocity. We also assume that too extreme depth differences between layers in the comfort zone could hamper a fast and accurate depth judgment. We plan to test whether more than two reference points (multiple depth layers) affect performance and could contribute to decrease the intersubjective variance of parallax boundaries. Finally, we aim at addressing the influence on the primary driving task through testing in a driving simulator.

\section{ACKNOWLEDGEMENTS}

The research leading to these results has received funding from the European Union Seventh Framework Programme ([FP7/20072013]) under grant agreement no. 244011 and 600851.

\section{REFERENCES}

[1] B. Alper, T. Hollerer, J. Kuchera-Morin, and A. Forbes. Stereoscopic highlighting: $2 \mathrm{~d}$ graph visualization on stereo displays. IEEE Transactions on Visualization and Computer Graphics, 17(12), 2011.

[2] N. Broy, E. André, and A. Schmidt. Is stereoscopic 3d a better choice for information representation in the car? In Proc. AutomotiveUI'12, New York, NY, USA, 2012. ACM.

[3] J. Cutting. How the eye measures reality and virtual reality. Behavior Research Methods, 29, 1997.

[4] G. E. Favalora. Volumetric 3d displays and application infrastructure. Computer, 38(8), 2005.

[5] B. Froner, N. S. Holliman, and S. P. Liversedge. A comparative study of fine depth perception on two-view $3 \mathrm{~d}$ displays. Displays, 29(5), 2008.

[6] G. Jones, D. Lee, N. Holliman, and D. Ezra. Controlling perceived depth in stereoscopic images. In Stereoscopic Displays and Virtual Reality Systems VIII, 2001.

[7] B. Julesz. Foundations of cyclopean perception. University of Chicago Press, 1971.

[8] M. Lambooij, M. Fortuin, I. Heynderickx, and W. IJsselsteijn. Visual discomfort and visual fatigue of stereoscopic displays: a review. Journal of Imaging Science, 2009.

[9] J. J. LaViola, Jr. and T. Litwiller. Evaluating the benefits of $3 \mathrm{~d}$ stereo in modern video games. In Proc. CHI'11, New York, 2011. ACM.

[10] K. Quintus and M. Halle. A composition tool for creating comfortable stereoscopic images. In Electronic Imaging 2008. International Society for Optics and Photonics, 2008.

[11] J. Schild, J. LaViola, and M. Masuch. Understanding user experience in stereoscopic 3d games. In Proc. CHI'12, New York, 2012. ACM.

[12] H. Snellen. Probebuchstaben zur Bestimmung der Sehschärfe. Nabu Press, 2010.

[13] W. Tam, F. Speranza, S. Yano, K. Shimono, and H. Ono. Stereoscopic 3D-TV: Visual Comfort. IEEE Transactions on Broadcasting, 57(2), 2011.

[14] M. Van Beurden, G. van Hoey, H. Hatzakis, and W. A. Ijsselsteijn. Stereoscopic displays in medical domains: A review of perception and performance effects. In Proc. SPIE'09, volume 7240, 2009.

[15] C. Ware. Information Visualization: Perception for Design. Interactive Technologies. Elsevier Science, 2004.

[16] M. Zwicker, W. Matusik, F. Durand, H. Pfister, and C. Forlines. Antialiasing for automultiscopic 3d displays. In ACM SIGGRAPH 'O6 Sketches, page 107. ACM, 2006. 